\title{
Pleiotropy method identifies genetic overlap between orofacial clefts at multiple loci from GWAS of multi-ethnic trios
}

Debashree Ray ${ }^{1,2, 凶}$, Sowmya Venkataraghavan ${ }^{1}$, Wanying Zhang ${ }^{1}$, Elizabeth J. Leslie ${ }^{3}$, Jacqueline B. Hetmanski ${ }^{1}$, Mary L Marazita ${ }^{4,5,6}$, Ingo Ruczinski ${ }^{2}$, Margaret A. Taub ${ }^{2}$ and Terri H. Beaty ${ }^{1,2, \bowtie}$

${ }^{1}$ Department of Epidemiology, Bloomberg School of Public Health, Johns Hopkins University, Baltimore, MD, USA.

${ }^{2}$ Department of Biostatistics, Bloomberg School of Public Health, Johns Hopkins University, Baltimore, MD, USA.

${ }^{3}$ Department of Human Genetics, School of Medicine, Emory University, Atlanta, GA, USA.

${ }^{4}$ Department of Oral Biology, School of Dental Medicine, University of Pittsburgh, PA, USA.

${ }^{5}$ Department of Human Genetics, Graduate School of Public Health, University of Pittsburgh, PA, USA.

${ }^{6}$ Center for Craniofacial and Dental Genetics, University of Pittsburgh, PA, USA.

${ }^{\circledR}$ Email: dray@jhu.edu; tbeaty1@jhu.edu

Based on epidemiologic and embryologic patterns, nonsyndromic orofacial clefts are commonly categorized into cleft lip with or without cleft palate (CL/P) and cleft palate alone (CP). While nearly forty risk genes have been identified for CL/P, few risk genes are known for CP. We used a new statistical method, PLACO, to identify genetic variants influencing risk of both CL/P and CP. In a combined multi-ethnic genome-wide study of 2,771 CL/P and $611 \mathrm{CP}$ case-parent trios, we discovered 6 new loci of genetic overlap between CL/P and CP; 3 new loci between pairwise OFC subtypes; and 4 loci not previously implicated in OFCs. We replicated the shared genetic etiology of subtypes underlying CL/P, and further discovered loci of genetic overlap exhibiting etiologic differences. In summary, we found evidence for 


\section{new genetic regions and confirmed some recognized $\mathrm{OFC}$ genes either exerting shared risk or with opposite effects on risk to OFC subtypes.}

\section{INTRODUCTION}

2 Orofacial clefts (OFCs) are the most common craniofacial birth defects that severely affect financial

3 and psychological well-being and the overall quality of life of the affected child and their family ${ }^{1}$.

4 These malformations most commonly occur as isolated defects (i.e., nonsyndromic clefts) and

5 affect, on average, nearly 1 out of 1,000 live births worldwide ${ }^{2}$. People born with OFCs require

6 multi-displinary medical treatments; have increased risk of psychological problems ${ }^{3}$; have greater

7 risk of various types of cancer (e.g., breast, brain and colon) ${ }^{4}$; and have increased mortality

8 throughout the life course ${ }^{5}$. Overall, OFCs pose a major public health burden, with underlying

9 biological mechanisms largely unknown.

Nonsyndromic OFCs typically manifest as a gap in the upper lip ('cleft lip' or CL) or the roof

11 of the mouth ('cleft palate' or CP) or both ('cleft lip and palate' or CLP). Based on epidemiologic

12 evidence, prevalence rates and the embryologic period when they develop, the subtypes CL and

13 CLP are typically grouped together as the subgroup CL/P (cleft lip with or without palate) ${ }^{6-8}$, while

$14 \mathrm{CP}$ alone forms the other subgroup. CL/P and CP have been historically analyzed separately ${ }^{9-12}$.

15 While genetic studies have identified nearly 40 genetic regions (or loci) as significantly associated

16 with risk to $\mathrm{CL} / \mathrm{P}$, fewer, around 10 loci, have been identified for $\mathrm{CP}^{2,13-15}$. The findings for $\mathrm{CP}$ have

17 mostly been identified in the Han Chinese population ${ }^{15}$. Together, these genetic regions explain no

18 more than a quarter of the estimated total heritability of risk to $\mathrm{OFCs}^{16}$.

19 Although the OFC subgroups CL/P and CP have been considered distinct, shared genetic

20 risk variants have been suggested ${ }^{9,11}$. There are multiplex cleft families with both CL/P and CP

21 present in affected relatives ${ }^{17,18}$. In recent years, there have been attempts to discover overlapping

22 genetic etiology of OFC subtypes. In this context, it is important to distinguish between genetic 
overlap and genetic heterogeneity. While genetic heterogeneity may refer to shared genetic effects as well as subtype-specific effects (which may mean a non-null effect on one subtype and no effect on the other), genetic overlap refers to non-null genetic effects on both subtypes that may or may not be equal in magnitude and/or direction. The usual approach for identifying genetic overlap in OFCs is to compare the significant findings from one subtype with those from the other ${ }^{11,13,19,20}$. However, the discovery of the associated variants in the first place may be under-powered in genome-wide association studies (GWAS) of each subtype separately. For instance, success of discovery genetics has been elusive for $\mathrm{CP}$, which could partially be due to smaller sample sizes of $\mathrm{CP}^{19}$ reflecting its lower birth prevalence. Another approach involves testing how well polygenic risk scores for one subtype can explain variation for another ${ }^{13,21}$, which describes overall genetic sharing, does not implicate specific regions of overlap (novel or otherwise), and may indicate lack of overlap when one subtype has a much smaller sample size ${ }^{13,14}$. One strategy is the "pooled method' GWAS analysis ${ }^{12}$, where all the OFC subtypes are pooled together in a combined analysis of all OFCs. FOXE1 has been successfully implicated as a shared risk gene using this approach ${ }^{12}$. While association signals from the pooled method may be driven by shared risk variants between subtypes, the pooled method does not necessarily capture only shared signals ${ }^{22}$, especially if sample sizes are widely different between the subtypes (e.g., the CL/P group is almost always much larger than the $\mathrm{CP}$ group) or if strong genetic effects exist in one group but not the other.

Furthermore, if a locus is hypothesized to have opposite genetic effects on the two subtypes (e.g., $\left.2 O G^{14,19}\right)$, the pooling technique will dilute any signal and consequently will be under-powered 3 to detect genetic overlap. Use of multi-trait methods in OFC genetic studies, such as the ones 4 commonly used in population-based GWAS of complex traits ${ }^{23-26}$, is hindered by the disjoint nature of the subtypes (i.e., absence of subjects with both traits), the qualitative (binary) nature 6 of the traits, and/or the case-parent trio design typically used to study multi-ethnic samples with 7 OFC. 
In this article, we use a new statistical method for pleiotropic analysis under composite null

$61 \&$ CP). hypothesis, PLACO, to discover genetic variants influencing risk of the two major nonsyndromic OFC subgroups (CL/P \& CP). Although PLACO was originally developed to discover pleiotropic variants between two traits from population-based studies ${ }^{27}$, we found it can also help identify genetic variants simultaneously influencing risk in two disease subgroups from family-based studies (see Methods). PLACO is particularly useful and powerful in identifying variants that increase risk of one subgroup while decreasing risk for the other, and seems to be robust to modest difference in sample sizes and in effect sizes between subgroups. We performed a meta-analysis GWAS using PLACO on 2,771 CL/P and $611 \mathrm{CP}$ multi-ethnic case-parent trios from the Pittsburgh Orofacial Cleft (POFC) and the Genes and Environment Association (GENEVA) studies. To dissect the genetic architecture at regions of genetic overlap between CL/P \& CP, we also explored genetic overlap stratified by racial/ethnic group, investigated if the overlapping genetic etiology is modified by sex, and explored if our findings are driven by specific pairs of OFC subtypes (CL \& CP or CLP

\section{RESULTS}

Identification of 9 loci with genetic overlap between CL/P \& CP, including 2 novel loci well-recognized risk gene that also happens to be a candidate shared gene ${ }^{28}$, 1q32.2 (IRF6, $p=$ $\left.4.3 \times 10^{-12}\right)$, while 2 loci in $1 \mathrm{p} 36.13\left(P A X 7, p=6.9 \times 10^{-8}\right)$ and $17 \mathrm{q} 22\left(\right.$ NOG, $\left.p=6.0 \times 10^{-8}\right)$

67 are suggestive, barely missing this significance threshold (Figure 1). Additionally, 6 loci showed 68 evidence for genetic overlap between CL/P \& CP at a suggestive threshold of $10^{-6}: 3 \mathrm{q} 29$ (DLG1, $\left.69 p=5.3 \times 10^{-7}\right), 4 \mathrm{p} 13\left(\right.$ LIMCH1, $\left.p=5.0 \times 10^{-7}\right), 4 \mathrm{q} 21.1\left(\right.$ SHROOM3, $\left.p=8.1 \times 10^{-7}\right), 9 \mathrm{q} 22.33$ $70\left(F O X E 1, p=1.7 \times 10^{-7}\right), 19 \mathrm{p} 13.12\left(R A B 8 A, p=6.8 \times 10^{-7}\right)$ and $20 \mathrm{q} 12\left(M A F B, p=9.9 \times 10^{-7}\right)$.

71 The 2 loci in $L I M C H 1$ and $R A B 8 A$ are novel for OFCs. All the other genes have been implicated 72 in GWAS of CL/P previously ${ }^{2,14}$, and insights into the molecular pathogenesis of OFCs via many 
73 of these genes is summarized elsewhere ${ }^{29}$. QQ plots from all our analyses show deviation from the

74 null only in the tail end of the distribution of p-values (Figures S1, S2), indicating genetic signals

75 rather than any systemic bias.

76 Six out of 9 loci yielding novel evidence for genetic overlap between CL/P \& CP. Of the 9

77 loci, genetic overlap at SNPs in/near genes $I R F 6^{28}, F O X E 1^{12}$ and $N O G^{19}$ have been previously

78 suggested in GWAS of clefts. We found novel, strong statistical evidence of genetic overlap at 79 the 6 loci in/near PAX7, DLG1, LIMCH1, SHROOM3, RABSA and MAFB. In particular, PLACO 80 provided stronger evidence for a pleiotropic association compared to the marginal association of 81 each subtype for these markers in DLG1, LIMCHI and RAB8A loci (Table 1).

82 Genetic sharing at these loci are not uniform in their effect on risk. We found the chosen 83 effect alleles at the lead SNPs in/near FOXE1, RAB8A and MAFB loci appear to increase risk for 84 both CL/P and CP, while the effect alleles at the remaining loci affect these OFC subgroups in 85 opposing directions as reflected by the estimated relative risks (RR) (Table 1). In other words, 86 the effect alleles at the lead SNPs at PAX7, IRF6, DLG1, LIMCH1, SHROOM3 and NOG seem to 87 predispose to one OFC subgroup while protecting from the other. The estimated RRs of the top 88 several SNPs at each of these loci further support this finding (Figure 2). In particular for markers 89 in the LIMCH1, IRF6, DLG1 and NOG loci, the estimated RRs of the lead SNP for subtypes $90 \mathrm{CL}$ and CLP (and hence CL/P) and their corresponding 95\% confidence intervals (CIs) were all 91 completely on the same side of the null value 1, while the estimated RR and its $95 \% \mathrm{CI}$ for $\mathrm{CP}$ 92 was completely on the other side (Figures 3, S3, S4, S5; panel b). The opposite effects of these 93 effect alleles likely explain why these loci were not conclusively identified as influencing risk to 94 both CL/P and CP in the 'pooled method' GWAS analysis of all OFC subtypes from POFC and 95 GENEVA subjects before ${ }^{12}$. Additionally, the IRF6 region appears to harbor at least 2 distinct loci: 96 one with shared genetic effects, and another with opposite effects (Figures 2, S6). Evidence for 
medRxiv preprint doi: https://doi.org/10.1101/2020.11.13.20231654; this version posted November 16, 2020. The copyright holder for this preprint (which was not certified by peer review) is the author/funder, who has granted medRxiv a license to display the preprint in perpetuity.

It is made available under a CC-BY-ND 4.0 International license .

97 both shared and opposite effects at IRF6 has been reported previously ${ }^{14,30}$.

98 Genetic overlap between subgroups CL/P \& CP is consistent with overlap identified between

99 pairwise OFC subtypes CL \& CP and CLP \& CP. To gain a better understanding of which

100 subtypes are driving this evidence of genetic overlap between CL/P \& CP, we applied PLACO on

101 the pairwise component OFC subtypes (Figure 4). The PAX7, SHROOM3, FOXE1 and MAFB loci

102 seem to be driven by the common genetic basis of subtypes CLP \& CP at these loci (Figures S7,

103 S8, S9, S10; panel g). The rest of the loci (LIMCH1, IRF6, DLG1, NOG and RAB8A) appear to

104 be driven by genetic overlap between CL \& CP as well as CLP \& CP (Figures 3, S3, S4, S5, S11;

105 panels $\mathbf{f}, \mathbf{g})$.

106

107

108

109

110

111

112

113

114

115

117

119

116 group. The regional association plots seem to indicate that signals of genetic overlap at the $P A X 7$,

118 the Asian subjects; while the $D L G 1$ and $R A B 8 A$ loci seem to draw upon evidence from both groups

Identification of additional novel regions of genetic overlap between component OFC subtypes. PLACO revealed 2 loci in 18q12.1 (MIR302F, $\left.p=6.2 \times 10^{-7}\right)$ and 10q24.33 (SH3PXD2A, $p=9.2 \times 10^{-7}$ ) associated with CL \& CP, and CLP \& CP subtypes respectively at a suggestive threshold of $10^{-6}$ (Table 1). Effect alleles at the lead SNPs of both these loci increase risk for one subtype while decreasing risk for the other (Figures S12, S13; panel b). The RR estimates and their 95\% CIs for the top several SNPs at these loci confirmed the opposite effect of these loci on risks of OFC subtypes (Figure 2). While $S H 3 P X D 2 A$ has been recently implicated in the formation of $\mathrm{CL} / \mathrm{P}$ in an European $\mathrm{GWAS}^{31}$, the $M I R 302 F$ locus is a novel genetic risk factor for OFCs.

15 Locus-specific effects at regions of genetic overlap between CL/P \& CP vary by racial/ethnic

17 FOXE1 and NOG loci are driven by the European subjects; IRF6, SHROOM3 and MAFB loci by

19 (Figures S3-S11; panels c-e). Similarly, evidence for the LIMCH1 locus seems to be driven by 
medRxiv preprint doi: https://doi.org/10.1101/2020.11.13.20231654; this version posted November $16,2020$. The copyright holder for this preprint (which was not certified by peer review) is the author/funder, who has granted medRxiv a license to display the preprint in perpetuity.

It is made available under a CC-BY-ND 4.0 International license .

120

121

122

123

124

125

126

127

128

129

130

131

132

133

134

135

136

137

138

139

140

141

142

143

both Asian and Latin American subjects (Figure 3; panels c, e). However, we must note that signals in these stratified analyses are confounded by differences in overall sample sizes between racial/ethnic groups (Table S1), sample size distribution between CL/P and CP subgroups, as well as minor allele frequency (MAF) differences across racial/ethnic groups. Consequently, the regional association plots do not fully indicate the differential information content of SNPs across these racial/ethnic groups. We, therefore, additionally provide a forest plot of RR estimates from the genotypic transmission disequilibrium test (gTDT) analyses stratified by cleft subtype and by racial/ethnic group for the lead SNP from each common locus (Figures 3, S3-S11; panel b). Notably for the Latin American subjects, the large uncertainty in the estimates for CP and the hugely skewed ratio of sample sizes between CL/P and CP are quite evident, leading to lack of power to drive signals of genetic overlap in this stratified analysis. The Asian and the European subgroups are more comparable in size, and findings from the regional association plots of these two racial/ethnic groups seem to be reflected in the forest plots as well.

\section{These regions of genetic overlap do not appear to be modified by sex. There is increasing} evidence for sex-specific differences in human health and disorders. While CL/P is 2 times more common in males, $\mathrm{CP}$ is more common in females ${ }^{2,29}$. Recent studies have indicated sex-specific differences in pleiotropic effects on complex traits ${ }^{32}$. We used PLACO to test for non-null SNP $\times$ Sex interaction effects in both CL/P \& CP, and failed to find any statistical evidence of pleiotropic effect modification by sex at the 9 loci of genetic overlap (Figure S14). We also did not find effect modification by sex at the 2 loci of genetic overlap, 18q12.1 and 10q24.33, identified between specific OFC subtypes. Tests of statistical interaction require larger sample sizes than tests of main effects; perhaps our sample size is not large enough to identify any effect modification by sex.

Proof of principle for sensitivity of PLACO in discovering shared etiology between OFC subtypes. Analysis of subtypes CL \& CLP using PLACO identified nearly all the recognized 
medRxiv preprint doi: https://doi.org/10.1101/2020.11.13.20231654; this version posted November 16 , 2020. The copyright holder for this preprint (which was not certified by peer review) is the author/funder, who has granted medRxiv a license to display the preprint in perpetuity.

It is made available under a CC-BY-ND 4.0 International license .

144 risk genes for CL/P subgroup alone as detected by the conventional gTDT analysis (Figure 5).

145 In other words, the regions of genetic overlap identified by PLACO matched the shared signals

146 captured by the pooled method analysis of CL and CLP subtypes. The RR estimates in Table

147 S2 indicate the effect alleles at lead SNPs in all regions of genetic overlap affect risk of both CL

148 and CLP in the same direction, which is consistent with the vast literature of epidemiologic and

149 genome-wide studies of $\mathrm{OFCs}^{6-8,12,14,20,30}$.

150 Beyond shared etiology, few loci of genetic overlap exhibit etiologic differences between

151 CL and CLP. When investigating the RR estimates from the top several SNPs at each of the

152 above-mentioned loci shared by CL and CLP, we found loci suggesting different pathogenesis

153 of these subtypes at 1p22.1 (ABCA4, ARHGAP29) and 1q32.2 (IRF6) as evidenced by a large

154 number of SNPs with opposite genetic effects (Figure S15). Some previous studies ${ }^{14,33}$ have noted

155 differences in genetic etiologies of CL and CLP, particularly at the IRF6 locus. Additionally,

156 the 1p36.13 (PAX7), 3q12.1 (COL8A1), 8q21.3 (DCAF4L2) and 8q24 (gene desert) regions with

157 shared effects between CL and CLP appear to have at least 2 distinct loci of genetic overlap

158 indicated by more than one peaks (Figure S16). Presence of possibly independent loci in the 8q24

159 region has been reported previously ${ }^{14}$. Furthermore, PLACO revealed a novel OFC locus at 1 p21.3

160 (MIR137HG, $p=2.2 \times 10^{-8}$ ) with opposite genetic effects for CL \& CLP at the genome-wide

161 threshold (Table 1 and Figure S17 a). The estimated RRs of the effect allele at the lead SNP

162 of this locus indicate its protective effect on CL, and its deleterious effect on subtypes CLP and

$163 \mathrm{CP}$ across racial/ethnic groups (Figure $\mathbf{S 1 7}$ b). This signal is, however, lost from CL/P (Figure

$164 \mathbf{S 1 7}$ c) due to pooling together of opposite effects of variants on CL and CLP (Figure S17 d, e).

165 Consequently, this locus near MIR137HG fails to show any evidence of genetic overlap between

166 CL/P \& CP (Figure S17 f) even though there is moderately strong statistical evidence of genetic

167 overlap between all pairs of OFC subtypes (Figure $\mathbf{S 1 7} \mathbf{a ,}, \mathbf{g}, \mathbf{h}$ ). 
medRxiv preprint doi: https://doi.org/10.1101/2020.11.13.20231654; this version posted November 16 , 2020. The copyright holder for this preprint (which was not certified by peer review) is the author/funder, who has granted medRxiv a license to display the preprint in perpetuity.

It is made available under a CC-BY-ND 4.0 International license .

168

169

170

171

172

173

174

175

176

177

178

179

180

181

182

183

184

185

186

187

188

189

190

191

192

\section{In-silico validation of robustness of PLACO to differences in sample sizes and modest subgroup-}

specific effects. To appreciate the advantages of PLACO and to interpret the following empirical results, it is important to briefly describe the intuition and statistical model behind PLACO. For two disease subgroups with genetic effects $\beta_{1}=\log \left(\mathrm{RR}_{1}\right)$ and $\beta_{2}=\log \left(\mathrm{RR}_{2}\right)$ for a variant, three possible situations can arise: global null, where the variant has no genetic effect on either subgroup $\left(\beta_{1}=0, \beta_{2}=0\right)$; sub-null, where the variant influences risk to one subgroup but not the other (either $\beta_{1}=0, \beta_{2} \neq 0$ or $\beta_{1} \neq 0, \beta_{2}=0$ ); and finally non-null, where the variant influences risk to both subgroups $\left(\beta_{1} \neq 0, \beta_{2} \neq 0\right)$. Only the non-null situation here describes genetic overlap between the two subgroups. PLACO tests a composite null hypothesis comprising both the global null and the sub-null situations, and thus rejection of this composite null provides statistical evidence of genetic overlap at a given variant (see Methods). On the other hand, the pooled method (previously used to identify risk variants common to both CL/P and CP) $)^{12}$ tests the global null hypothesis, and it may be rejected because either the sub-null or the non-null situations exist.

We compared PLACO with the pooled method across multiple simulation scenarios. When almost all of the simulated null variants had no effect on either OFC subgroup (Scenario I majority of variants under the global null situation), both PLACO and the pooled method showed well-controlled type I error rates (Figure S19 a). As the sample sizes became skewed between the OFC subgroups, the pooled method showed inflated type I error while PLACO maintained appropriate type I error rate even at stringent error levels (Figure S19 b-c). When a large proportion of simulated null variants had a genetic effect on one OFC subgroup only (Scenario II - majority of variants under the sub-null situation), the pooled method had hugely inflated type I error while PLACO showed proper type I error control at stringent levels regardless of skewed sample sizes between the two OFC subgroups (Figure S20 a-c). This observation holds true irrespective of how widely different the MAFs are between the two simulated ethnic groups (Figure $\mathbf{S 2 0}$ d-f). This 
medRxiv preprint doi: https://doi.org/10.1101/2020.11.13.20231654; this version posted November $16,2020$. The copyright holder for this preprint (which was not certified by peer review) is the author/funder, who has granted medRxiv a license to display the preprint in perpetuity.

It is made available under a CC-BY-ND 4.0 International license .

193 shows how the pooled method may show spurious signals (or increased false discoveries) if genetic

194 effect exists in one OFC subgroup but not the other, and if there is a large sample size difference

195 between subgroups. On the other hand, our empirical results suggest robustness of PLACO's type

196 I error to sample size differences between OFC subgroups; moderately strong subgroup-specific

197 effects; and small to large MAF differences between ethnic groups.

198 Empirical evidence of sensitivity of PLACO in discovering common genetic basis of OFC

199 subgroups. We benchmarked the power of PLACO against the pooled method (even though 200 pooled method shows increased false discoveries under the sub-null situations) along with the 201 naive approach of declaring genetic overlap when a variant reaches genome-wide significance for 202 the larger OFC subgroup (in our case, CL/P) and reaches a more liberal significance threshold 203 for the other. We used two such naive approaches: one based on the criterion $p_{\mathrm{CL} / \mathrm{P}}<5 \times 10^{-8}$, $204 p_{\mathrm{CP}}<10^{-5}$ and the other $p_{\mathrm{CL} / \mathrm{P}}<5 \times 10^{-8}, p_{\mathrm{CP}}<10^{-3}$ ('Naive-1' and 'Naive-2' respectively in 205 our figures). Regardless of the magnitude and directions of genetic effect on these OFC subgroups, 206 and the sample size differences, PLACO showed dramatically improved statistical power to detect 207 common genetic basis compared to the naive approaches (Figure S21). For instance, the 'Naive-2' 208 method with a liberal threshold criterion has a $24 \%$ power, compared to $61 \%$ for PLACO, to 209 detect simultaneous association using 1,800 CL/P and $600 \mathrm{CP}$ trios when an MAF 10\% variant 210 influences risk to both $\mathrm{CL} / \mathrm{P}$ and $\mathrm{CP}$ with $\mathrm{RR}=1.5$. This probably explains why a genome-wide 211 analysis of CL/P first, followed by an analysis of CP on the most significant findings, have not 212 quite proven successful in providing evidence of overlapping association signals between these 213 two OFC subgroups. Although PLACO was slightly less powerful than the pooled method in 214 identifying shared risk variants, it did achieve greater power gain when detecting variants that 215 increased risk for one OFC subgroup while decreasing risk for the other (Figure S21). 


\section{DISCUSSION}

217 In this analysis of multi-ethnic case-parent trios from the POFC and the GENEVA studies, we

218 identified genetic overlap between nonsyndromic CL/P \& CP at 1 locus in 1q32.2 reaching genome-wide

219 significance $\left(5 \times 10^{-8}\right), 2$ loci in 1 p36.13 and $17 \mathrm{q} 22$ barely missing this conventional threshold, 220 and 6 loci in 3q29, 4p13, 4q21.1, 9q22.33, 19p13.12 and 20q12 yielding suggestive significance 221 at a threshold of $10^{-6}$. The apparent risk SNPs at 4p13 and 19p13.12 are located in the LIMCH1

222

223

224

225

226

227

228

229

230

231

232

233

234

235

236

237

238

239 alterations of the SHROOM3 gene ${ }^{38}$, and $M A F B$ has been identified in GWAS of CLP with mouse

240 models showing its role in palate development ${ }^{29}$. 
The associated SNPs at the 4p13 locus occur within a topologically associating domain 242 containing two genes: LIMCH1 and $U C H L 1^{39}$. This locus was previously found to be suggestively 243 associated with CL/P using GENEVA and POFC subjects ${ }^{14}$. To our knowledge, neither of these 244 genes has been directly implicated in development of clefts to date. There is some evidence of 245 altered methylation patterns in peripheral blood for $\mathrm{LIMCH} 1$ found in Han Chinese pedigrees with children affected by NTDs ${ }^{40}$. Although OFCs and NTDs are considered 'mid-line birth defects', and supplementing mothers with folate and multivitamins during pregnancy seem to reduce risk to

248

249 250 251 252 253 254 255 256 257 258 259 260 261 262 263 264 265 both $^{41}$, it remains unknown if the same genes influence their risk. The region around the associated SNPs contains multiple putative craniofacial enhancers derived from epigenomic marks in human fetal craniofacial tissue, and the $L I M C H 1$ and $U C H L 1$ genes are decorated with marks associated with active transcription ${ }^{42}$. These data suggest that this locus could play a role in craniofacial development but provide no clues for the opposite effects of SNPs on the risk to CL/P and CP.

Similarly, SH3PXD2A was recently implicated in the etiology of CL/P for the first time in a GWAS of individuals from the Netherlands and Belgium ${ }^{31}$. Zebrafish and mouse models support some role of this gene in $\mathrm{OFCs}^{31}$. Homozygous disruption of this gene in mutant mice resulted in complete clefts of the secondary palate ${ }^{43}$. These studies suggest that $S H 3 P X D 2 A$ might play a role in the pathogenesis of both CL/P and CP, but it is not yet clear how opposite genetic effects of the markers near this gene mechanistically influence risk to subtypes CLP and CP.

We also found opposite effects of associated SNPs for CL and CP near another novel OFC gene, $M I R 302 F$. There is some evidence that MIR302 family members regulate TP63, a gene found mutated in ectodermal dysplasia-clefting syndrome ${ }^{29}$. In mouse models, members of the miR-302 family (miR-302 a-d) target different isoforms of the p63 transcription factor, the expression of which is critical for normal lip and palate development. Complete loss of p63 expression leads to CLP in mouse models ${ }^{44}$. Unfortunately, these studies did not include miR-302f, and little is known about the $M I R 302 F$ gene. It is possible that $M I R 302 F$ plays a similar critical role in craniofacial 
development as the other members of the MIR302 family.

$$
\text { In this manuscript we have annotated the 1p21.3 and 19p13.12 loci with the genes MIR } 137 H G
$$
and $R A B 8 A$, respectively. However, these annotations are based on proximity to the most significant SNPs and there is no specific evidence in the literature to support their role in craniofacial development. There are 3 genes in the topological domain containing the associated SNPs of 1p21.3 locus (MIR137HG, MIR2682 and DPYD) ${ }^{39}$, which may be associated with schizophrenia and bipolar disorder ${ }^{45}$. RAB8A itself has been shown to be associated with endometrial cancer ${ }^{46}$. It will be an area of future work to replicate and further elucidate our findings near RAB8A and MIR137HG.

Taken together, our study provides strong statistical evidence for possible overlap in the genetic architectures of CL/P and CP. Historically, these two OFC subgroups have been thought to have distinct etiologies based on developmental origins and epidemiologic patterns. Linkage studies first identified significant evidence of linkage for markers in the FOXE1 region in CL/P multiplex families ${ }^{47}$; subsequent studies confirmed this gene as a risk factor for both CL/P and $\mathrm{CP}^{11,48}$. Linkage analysis identified a susceptibility locus near $T B X 22$ for CL/P and a later study found mutations in $T B X 22$ in CP individuals ${ }^{17}$. Fine-mapping of translocation breakpoints revealed an important role of $S A T B 2$ in cases with $\mathrm{CP}$; a few years later, a candidate gene study identified significant association of a variant in SATB2 with CL/P in two Asian populations ${ }^{10,17}$. There exists some evidence that variants in $I R F 6^{28}, G R H L 3^{28}, A R H G A P 29^{49-51}$ and $M S X 1^{17}$ regions may affect risk to both $\mathrm{CL} / \mathrm{P}$ and $\mathrm{CP}$ in the same direction (often termed as 'shared genetic risk'). Note, much of this evidence are from patterns of Mendelian inheritance of rare variants in extended pedigrees; genetic overlap of common variants in nonsyndromic OFCs may not follow the same patterns. In recent GWAS, variants near $I R F 6^{20}$ and $N O G^{14,19}$ have shown weak evidence of decreased risk for one OFC subgroup and increased risk for the other. Among these well-recognized risk genes for OFCs, only FOXE1 has been successfully implicated as a shared risk gene in GWAS ${ }^{12}$. Our method PLACO not only replicated this finding for FOXE1, but also provided strong statistical evidence for 
291 292 293

294 295

genetic overlap at IRF6 and NOG. PLACO found no evidence of genetic overlap between CL/P \& CP at common variants (MAF $\geq 5 \%$ ) in/near SATB2 (chr2:200134004-200316268, including rs6705250 and rs12105015; $\left.p_{\min }=0.03\right)$, GRHL3, ARHGAP29 or MSX1 (Figure S18). It is possible that rarer variants drive genetic overlap in these regions, and PLACO is currently only applicable to common variants.

In summary, this study advanced our knowledge of the genetic architecture controlling risk to OFCs by enriching the current inventory of OFC-associated genes with novel genes possibly driving common genetic basis of OFC subtypes. Lack of granularity of cleft subtype in animal models precludes experimental validation of our findings. No bioinformatics analysis on existing large-scale databases is equipped to explain how some of these loci could affect the two OFC subgroups in opposing directions. Instead we utilized in-silico validation techniques such as statistical simulation experiments, sensitivity analyses, and proof-of-principle analysis. Our extensive in-silico validation showed PLACO's robustness to subgroup-specific effects (not a situation of genetic overlap), population-specific differences in MAF, and sample size differences between OFC subgroups. Our proof-of-principle analysis of subtypes CL \& CLP using PLACO and replicating those findings with genetic associations of subgroup CL/P emphasized the shared etiology successfully identified by PLACO. More granular functional studies than those currently available are needed to clearly understand the differences in how some of the genes identified here could affect risk of one subtype versus another.

\section{METHODS}

The POFC and the GENEVA studies. Case-parent trios ascertained through cases with an isolated, nonsyndromic OFC in the GENEVA study were largely recruited through surgical treatment centers by multiple investigators from Europe (Norway and Denmark), the United States (Iowa, Maryland, Pennsylvania, and Utah) and Asia (People's Republic of China, Taiwan, South Korea, 
315 Singapore, and the Philippines) $)^{12,49}$. Type of cleft, sex, race as well as common environmental risk

316 factors were obtained through direct maternal interview ${ }^{49}$. The research protocol was approved by

317 the Institutional Review Boards (IRB) at the Johns Hopkins Bloomberg School of Public Health

318 and at each participating recruitment site. Written informed consent was obtained from both

319 parents and assent from the child was solicited whenever the child was old enough to understand

320 the purpose of the study.

321 The POFC study included case-parent trios ascertained through a proband with an isolated 322 nonsyndromic $\mathrm{CL} / \mathrm{P}$ or $\mathrm{CP}$ from multiple populations, and a large number of OFC cases and 323 ethnically matched controls from some of these same populations ${ }^{12,52}$. We, however, used only 324 the case-parent trios from POFC in this study. Similar information about type of cleft, sex, race 325 and common environmental risk factors were collected through direct maternal interview. The 326 research protocol was approved by the IRB at the University of Pittsburgh and all participating 327 institutions, and informed consent was obtained from all participants.

The distribution of trios by cleft subtype and racial/ethnic groups from both studies is given in

329 Table S1. It is to be noted that originally 412 individuals from POFC were included in GENEVA ${ }^{52}$; 330 we have subsequently removed them from our GENEVA dataset to avoid duplication. Thus, in 331 this article, these two studies represent independent, non-overlapping case-parent trios from three 332 major racial/ethnic groups (European, Asian, and Latin American). Instead of having separate 333 discovery and replication samples, we decided to combine the two studies, which should have 334 improved power to detect genetic associations over a two-stage discovery-replication approach ${ }^{53}$.

335 Genotyping, imputation and quality control. Participants in the GENEVA study were genotyped 336 on the Illumina Human610 Quadv1_B array with 589,945 SNPs at the Center for Inherited Disease 337 Research (https://cidr.jhmi.edu/). We re-imputed this dataset using the Michigan Imputation Server ${ }^{54}$ 338 to take advantage of more efficient imputation tools and more recent, larger reference panels. 
339 Before imputing, we dropped SNPs with $\mathrm{MAF}<1 \%$, performed trio-aware phasing of the haplotypes

340 from the observed genotypes using SHAPEIT2 $2^{55}$, and original genotyped SNPs on build hg 18 were

341 lifted over to hg19 (http://github.com/sritchie73/liftOverPlink). We used the 1000 Genomes Phase

3423 release 5 reference panel for imputation. Note, trio-aware phasing before imputation is critical;

343 ignoring the family information may lead to biased downstream results ${ }^{56}$. 'Hard' genotype calls

344 were made by setting threshold 0.1 within PLINK 2.0 (www.cog- genomics.org/plink/2.0/) ${ }^{57}$. If

345 the calls have uncertainty $>0.1$ (i.e., genotype likelihoods $<0.9$ ), they were treated as missing; the

346 rest were regarded as hard genotype calls. We took the following quality control measures: all

347 genotyped SNPs with missingness $>5 \%$, Mendelian error rate $>5 \%$, all SNPs with MAF $<5 \%$ as

348 well as those showing deviation from Hardy-Weinberg equilibrium (HWE) at $p<10^{-4}$ among

349 parents were dropped. All imputed SNPs were filtered to exclude any with $R^{2}<0.3$ using

350 BCFtools-v1.9 (https://samtools.github.io/bcftools). Additionally, individuals with low genotype

351 information or evidence of low-quality DNA, individuals with SNP missingness $>10 \%$, and individuals

352 duplicated across the POFC and GENEVA datasets were excluded. Only complete trios were

353 kept for the final analysis. The final GENEVA dataset contained 6,762,077 autosomal SNPs,

354 including both observed and imputed SNPs having MAF $\geq 5 \%$ among parents, for 1, 939 complete

355 case-parent trios.

356 The case-parent trios from the POFC study were genotyped on 539,473 SNPs on the Illumina

357 HumanCore + Exome array. For imputation, data were phased with SHAPEIT2 and imputed using

358 IMPUTE2 $^{58}$ to the 1000 Genomes Phase 3 reference panel as described previously ${ }^{52}$. Incomplete

359 trios, trios with parents from different racial/ethnic groups and racial/ethnic groups with insufficient

360 sample sizes for effective imputation were dropped. The same quality control measures as GENEVA

361 were used to remove rare and poor quality SNPs. The final POFC dataset contained 6, 350, 243

362 autosomal SNPs, including both observed and imputed SNPs having MAF $\geq 5 \%$ among parents,

363 for 1, 443 complete case-parent trios. 
We meta-analyzed the POFC and the GENEVA studies (which are independent) to increase sample size and power. All SNPs with mismatch in allele or base pair information (hg19) between

366 the two studies were removed. The final meta-analyzed dataset contained 6, 761, 961 SNPs, which

367 includes all SNPs common to the two studies and SNPs unique to any one study.

368 PLACO: pleiotropic analysis under a composite null hypothesis. Consider genome-wide studies

369 of two disorders $Y_{1}$ and $Y_{2}$ based on $n_{1}$ and $n_{2}$ case-parent trios respectively who were genotyped/ 370 imputed or sequenced at $p$ SNPs. For a given SNP, assume an additive genetic model where the 371 relative risks associated with the two disorders are $R_{1}$ and $R_{2}$. The corresponding genetic 372 effect parameters are respectively $\beta_{1}=\log \left(R_{1}\right)$ and $\beta_{2}=\log \left(R_{2}\right)$. One may assume any 373 other genetic inheritance model, and the following is still applicable. In each study, the genotypic 374 transmission disequilibrium test (gTDT) using a conditional logistic framework ${ }^{59,60}$ may be used to 375 obtain the maximum likelihood estimates $\hat{\beta}_{k}$ and its standard error $\hat{\mathrm{se}}_{k}$, which are used to construct 376 the summary statistic $Z_{k}=\frac{\hat{\beta}_{k}}{\hat{\mathrm{s}}_{k}}$ for $k=1,2$. Since TDTs for trios protect against confounding 377 due to population stratification, one may combine multi-ethnic case-parent trios when analyzing 378 the two disorders.

To implement PLACO, we start with the summary statistics $Z_{1}$ and $Z_{2}$ from the two disorders across all SNPs genome-wide. For practical purposes, we assume the datasets for the two disorders are independent since case-parent trios are ascertained based on the disease status of the child and it is unlikely to have subjects shared between the two datasets. While the usual multi-trait methods $^{23,24}$ test the null hypothesis of no association of a given SNP with any disorder (i.e., $\beta_{1}=0=\beta_{2}$ ) against the alternative hypothesis that at least one disorder is associated, PLACO tests the composite null hypothesis that at most one disorder is associated with the SNP against the alternative that both disorders are associated ${ }^{27}$. Mathematically, PLACO tests

$$
H_{0}: \beta_{1} \beta_{2}=0 \text { versus } H_{a}: \beta_{1} \beta_{2} \neq 0
$$


so that rejection of the null hypothesis statistically indicates genetic overlap between disorders. The null hypothesis $H_{0}$ is a composite of the global null $\left\{\beta_{1}=0=\beta_{2}\right\}$, and the sub-nulls $\left\{\beta_{1}=0, \beta_{2} \neq 0\right\}$ and $\left\{\beta_{1} \neq 0, \beta_{2}=0\right\}$. Suppose, across the genome, the global null holds with probability $\pi_{00}$ under which the summary statistics $Z_{1}$ and $Z_{2}$ have asymptotic standard normal distributions. Further assume that the first sub-null holds with probability $\pi_{01}$ where $Z_{1}$ has a standard normal distribution and $Z_{2}$ has a shifted normal distribution $N\left(\mu_{2}, 1\right)$. For a given disorder, the relative risks of SNPs with a non-null effect vary genome-wide. Consequently, there is no fixed value that the mean parameter $\mu_{2}$ takes, and to capture this variability in effect sizes we assume a random effect on the mean - a $N\left(0, \tau_{2}^{2}\right)$ distribution. Similarly, assume that the second sub-null holds with probability $\pi_{02}$ and $Z_{2} \sim N(0,1)$ while $Z_{1}$ given $\mu_{1}$ has a $N\left(\mu_{1}, 1\right)$ distribution, where $\mu_{1}$ is assumed to follow a $N\left(0, \tau_{1}^{2}\right)$ distribution.

Thus, under the composite null hypothesis $H_{0}$, PLACO assumes (a) $Z_{1}$ and $Z_{2}$ are independent $N(0,1)$ variables when $\left\{\beta_{1}=0, \beta_{2}=0\right\}$ holds; (b) $Z_{1}$ and $Z_{2}$ are independent $N(0,1)$ and $N\left(0,1+\tau_{2}^{2}\right)$ variables respectively when $\left\{\beta_{1}=0, \beta_{2} \neq 0\right\}$ holds; and (c) $Z_{1}$ and $Z_{2}$ are independent $N\left(0,1+\tau_{1}^{2}\right)$ and $N(0,1)$ variables respectively when $\left\{\beta_{1} \neq 0, \beta_{2}=0\right\}$ holds. We have described the rationale and other considerations behind this choice of PLACO model previously ${ }^{27}$. The PLACO test statistic is

$$
T_{\mathrm{PLACO}}=Z_{1} Z_{2}
$$

and its approximate, asymptotic p-value is given by

$$
p_{\text {PLACO }}=\mathbb{F}\left(z_{1} z_{2} / \sqrt{\operatorname{Var}\left(Z_{1}\right)}\right)+\mathbb{F}\left(z_{1} z_{2} / \sqrt{\operatorname{Var}\left(Z_{2}\right)}\right)-\mathbb{F}\left(z_{1} z_{2}\right)
$$

390 where $z_{1}$ and $z_{2}$ are the observed $Z$-scores for the two disorders at any given $\operatorname{SNP} ; \mathbb{F}(u)=$ $3912 \int_{|u|}^{\infty} \mathbb{f}(x) d x$ is the two-sided tail probability of a normal product distribution at value $u$; and $392 \operatorname{Var}\left(Z_{1}\right)$ and $\operatorname{Var}\left(Z_{2}\right)$ are the estimated marginal variances of these $Z$-scores under the above 393 distributional mode ${ }^{27}$. Open-source implementation of PLACO in $\mathrm{R}^{61}$ is available at https: 
394 395 396 397 398 399 400 401 402 403 404 405 406 407 408 409

//github.com/RayDebashree/PLACO. While PLACO was originally proposed for two traits from population-based studies (e.g. case-control traits) ${ }^{27}$, here we showed PLACO can very well be used for family studies (Supplementary S2) as long as the summary statistics are obtained after appropriately accounting for all confounding effects, including relatedness and population stratification.

Statistical analyses. For all analyses presented here, we focused on bi-allelic SNPs with MAF $\geq 5 \%$, where MAF is calculated based on only the parents (founders) using PLINK 2.0. For each study separately, we obtained summary statistics of genetic association between each variant and each OFC subgroup using the gTDT model for case-parent trios under an additive genetic model as implemented in R package $\operatorname{trio}^{59}$ (v3.20.0). We used the gTDT over the allelic TDT because of its several advantages ${ }^{62}$ : gTDT can be more powerful; yields parameter estimates, standard errors along with p-values; and enables direct assessment of RR. However, unlike the allelic TDT ${ }^{63}$, the gTDT assumes a specific mode of inheritance; here we chose an additive model. The gTDT in trio package uses the minor allele of the input dataset as the effect allele. Since the gTDT is applied on each subgroup and each study separately, it is possible that a minor allele is not the same across subgroups and across studies. Therefore, we set the minor allele from the CL/P subgroup in POFC as the effect allele for all analyses. We, then, meta-analyzed the gTDT results over the two studies using inverse-variance weighted fixed effects meta-analysis. We implemented PLACO on the meta-analyzed gTDT results from subgroups $\mathrm{CL} / \mathrm{P}$ and $\mathrm{CP}$ to identify possible genetic overlap between them. To identify regions of significant genetic overlap, we used the conventional genome-wide threshold $5 \times 10^{-8}$ and also a suggestive threshold of $10^{-6}$.

We explored if any identified region of genetic overlap was modified by sex. To do this, we first obtained summary statistics from the $1 \mathrm{df} \mathrm{SNP} \times \mathrm{Sex}$ analysis using the gene-environment gTDT model in trio package (again assuming additive genetic model); then meta-analyzed the 
$418 \mathrm{SNP} \times$ Sex estimates across the two studies; and finally applied PLACO on the meta-analyzed $1 \mathrm{df}$ $419 \mathrm{SNP} \times$ Sex summary statistics. For each analysis, we created Manhattan plots to show signals, and 420 QQ plots to check for potential bias in the association results. For the QQ plots, we also calculated 421 the genomic inflation factors at the $50^{\text {th }}$ percentile $\left(\lambda_{0.5}\right)$ to quantify the extent of the bulk inflation, 422 and at the $1 / 10^{\text {th }}$ of a percentile $\left(\lambda_{0.001}\right)$ to quantify inflation towards the meaningful tail of the 423 distribution. We took the p-values from a given method (e.g., gTDT, PLACO), mapped them to 1 424 df $\chi^{2}$ statistics, and calculated $\lambda_{x}$ as the ratio of empirical $100(1-x)^{\text {th }}$ percentile of these statistics 425 and the theoretical $100(1-x)^{\text {th }}$ percentile of $1 \mathrm{df} \chi^{2}$ distribution.

Stratified analyses. We considered two stratified analyses: one stratified by racial/ethnic group and the other by cleft subtype (CL, CLP, CP). As described before, genome-wide meta-analyzed gTDT summary statistics were obtained for CL/P and for $\mathrm{CP}$ within each of the three major racial/ethnic groups: European, Asian and Latin American. PLACO was applied on CL/P and CP summary data within each racial/ethnic group. For the OFC subtype stratified analyses, meta-analyzed gTDT summary statistics were obtained for each cleft subtype, and then PLACO was applied on each of the three pairwise combinations to compare and contrast results against those from the main analysis.

Locus annotation and candidate gene prioritization. For each analysis of genetic overlap, we defined independent loci by clumping all the suggestively significant SNPs $\left(p_{\text {PLACO }}<10^{-6}\right)$ in $\mathrm{a} \pm 500 \mathrm{~Kb}$ radius and with linkage disequilibrium (LD) $r^{2}>0.2$ into a single genetic locus.

37 This clumping was done using FUMA $^{64}$ (SNP2GENE function, v1.3.5e). Since we performed

38 multi-ethnic analysis, we separately used 1000G Phase 3 EUR, EAS and AMR as reference populations

39 for LD calculation. The number of independent loci and the index SNP for each locus (chosen to 40 be the most significant SNP) were the same regardless of the racial/ethnic groups assumed for LD calculation. To define the bounds of each locus, as used in the regional association plots annotated 
442 by effect size directions (Figures S6, S16 and S17 e), we took the minimum of lower bounds and 443 the maximum of upper bounds across racial/ethnic groups. We mapped each locus to the gene 444 nearest to the lead SNP using FUMA. We used LocusZoom ${ }^{65}$ to get regional association plots with 445 gene tracks that allowed us to examine detailed evidence of association at each identified locus. 446 For these LocusZoom plots, we used genome build hg19 with no specified LD reference panel due 447 to the multi-ethnic nature of our analysis. The LocusZoom plots for the stratified analyses of the 448 three racial/ethnic groups, however, use the corresponding LD reference panel.

449 Validation. As mentioned before, we do not have separate discovery and validation samples; 450 combining samples improves power to detect genetic associations over a two-stage discovery451 replication approach ${ }^{53}$. Experimental validation in animal models is not possible due to the lack 452 of granularity of cleft subtypes in mouse or zebrafish models. No current bioinformatics analysis 453 can fully explain the opposite effects of the loci discovered here (where the effect allele increases 454 risk for one OFC subgroup but decreases risk for another). To provide confidence on PLACO's 455 findings, we undertook three complementary approaches: (1) a proof-of-principle analysis of 456 subtypes CL \& CLP using PLACO and matching those findings with genetic associations of 457 subgroup CL/P to emphasize the shared etiology that PLACO successfully identified; (2) an in-silico 458 validation of PLACO using simulated data; and (3) an assessment of our findings based on existing 459 literature. In particular, our extensive empirical validation involves showing (i) PLACO's robustness 460 to subgroup-specific effects, population-specific differences in MAF, and sample size differences 461 between OFC subgroups; and (ii) massive power gains achieved by PLACO in detecting genetic 462 overlap (whether shared or in opposing directions) compared to other commonly-used variant-level 463 approaches.

464 In-silico evaluation of PLACO using simulated data for OFC trios. We simulated two bi-ethnic 465 case-parent trio datasets with a total of 2, 400 trios mimicking independent studies of CL/P and 
medRxiv preprint doi: https://doi.org/10.1101/2020.11.13.20231654; this version posted November 16, 2020. The copyright holder for this preprint (which was not certified by peer review) is the author/funder, who has granted medRxiv a license to display the preprint in perpetuity.

It is made available under a CC-BY-ND 4.0 International license .

$466 \mathrm{CP}$. We assumed, without loss of generality, that the two ethnic groups have equal sample sizes

467 for a particular OFC subgroup, and considered situations where the OFC subgroups either have 468 comparable $(1: 1)$ or unbalanced $(3: 1)$ or largely unbalanced $(7: 1)$ sample sizes similar to what we 469 saw for the POFC and the GENEVA studies. We simulated the two ethnic groups such that they 470 are different in terms of OFC subgroup prevalence, and in terms of MAF at any given variant. We 471 compared type I error and power of PLACO with the pooled method and the naive approach of 472 declaring genetic overlap when a variant reaches genome-wide significance for the subgroup with 473 the larger sample size and reaches a more liberal significance threshold for the second subgroup. 474 See Supplementary S2 for more details on our simulation experiments.

Data availability. The POFC and the GENEVA studies are publicly available on dbGaP (https://www.ncbi.nlm.nih.gov/gap/, study accession numbers phs000774.v1.p1 and phs000094.v1.p1).

Genome build. All genomic coordinates are given in NCBI Build 37/UCSC hg19.

Web ReSOURCES The R source code for the genetic overlap test PLACO can be found in GitHub (https://github.com/RayDebashree/PLACO/). R trio package can be found in Bioconductor (https://bioconductor.org/packages/release/bioc/html/trio.html).

SupPlemental Data Supplementary information is available on the journal website.

ACKNOWLEDGMents This research was supported in part by the NIH grants R03DE029254 (D.R., S.V., J.B.H., T.H.B.), R03DE027121 (S.V., W.Z., M.A.T., T.H.B.), R00DE025060 (E.J.L.), R01DE016148 (M.L.M.) and U24OD023382 (D.R.). It was carried out using computing cluster - the Joint High Performance Computing Exchange - at the Department of Biostatistics, Johns Hopkins Bloomberg School of Public Health.

Author Contributions D.R., M.A.T. and T.H.B. designed this study and performed overall project management. W.Z. and J.B.H. performed imputation and processing of the GENEVA data. D.R., S.V. 
medRxiv preprint doi: https://doi.org/10.1101/2020.11.13.20231654; this version posted November 16, 2020. The copyright holder for this preprint (which was not certified by peer review) is the author/funder, who has granted medRxiv a license to display the preprint in perpetuity.

It is made available under a CC-BY-ND 4.0 International license .

and W.Z. analyzed the POFC and the GENEVA data. D.R., E.J.L., I.R., M.A.T. and T.H.B. interpreted the scientific findings. D.R. and S.V. performed statistical simulation experiments. M.L.M. and T.H.B. provided the POFC and the GENEVA data respectively. D.R. drafted the initial manuscript. All authors reviewed and approved the final manuscript.

COMPETING FINANCIAL INTERESTS The authors declare no competing financial interests.

CORRESPONDENCE Correspondence should be addressed to D.R. (email: dray@jhu.edu) or T.H.B. (email: tbeaty1@jhu.edu).

\section{REFERENCES}

1. Wehby, G. \& Cassell, C. H. The impact of orofacial clefts on quality of life and healthcare use and costs. Oral Dis 16, 3-10 (2010).

2. Beaty, T. H., Marazita, M. L. \& Leslie, E. J. Genetic factors influencing risk to orofacial clefts: today's challenges and tomorrow's opportunities. F1000Research 5 (2016).

3. Christensen, K. \& Mortensen, P. B. Facial clefting and psychiatric diseases: a follow-up of the Danish 1936-1987 Facial Cleft cohort. Cleft Palate Craniofac J 39, 392-396 (2002).

4. Leslie, E. J. \& Marazita, M. L. Genetics of cleft lip and cleft palate. Am J Med Genet Part C Semin Med Genet 163C, 246-258 (2013).

5. Christensen, K., Juel, K., Herskind, A. M. \& Murray, J. C. Long term follow up study of survival associated with cleft lip and palate at birth. BMJ 328, 1405 (2004).

6. Sperber, G. H. in Cleft lip and palate: from origin to treatment (ed Wyszynski, D. F.) 5-24 (Oxford University Press, Oxford, 2002).

7. Sivertsen, Å. et al. Familial risk of oral clefts by morphological type and severity: population based cohort study of first degree relatives. BMJ 336, 432-434 (2008). 
8. Mangold, E., Ludwig, K. U. \& Nöthen, M. M. Breakthroughs in the genetics of orofacial clefting. Trends Mol Med 17, 725-733 (2011).

9. Kondo, S. et al. Mutations in IRF6 cause Van der Woude and popliteal pterygium syndromes. Nat Genet 32, 285-289 (2002).

10. Beaty, T. et al. Analysis of candidate genes on chromosome 2 in oral cleft case-parent trios from three populations. Hum Genet 120, 501-518 (2006).

11. Moreno, L. M. et al. FOXE1 association with both isolated cleft lip with or without cleft palate, and isolated cleft palate. Hum Mol Genet 18, 4879-4896 (2009).

12. Leslie, E. J. et al. Genome-wide meta-analyses of nonsyndromic orofacial clefts identify novel associations between FOXE1 and all orofacial clefts, and TP63 and cleft lip with or without cleft palate. Hum Genet 136, 275-286 (2017).

13. Ludwig, K. U. et al. Imputation of orofacial clefting data identifies novel risk loci and sheds light on the genetic background of cleft lip \pm cleft palate and cleft palate only. Hum Mol Genet 26, 829-842 (2017).

14. Carlson, J. C. et al. A systematic genetic analysis and visualization of phenotypic heterogeneity among orofacial cleft GWAS signals. Genet Epidemiol 43, 704-716 (2019).

15. He, M et al. Genome-wide analyses identify a novel risk locus for nonsyndromic cleft palate. J Dent Res 10, 1-8 (2020).

16. Marazita, M. \& Leslie, E. in Comprehensive cleft care (eds Losee, J. \& Kirschner, R.) 207-224 (CRC Press, Boca Raton FL, 2016).

17. Rahimov, F., Jugessur, A. \& Murray, J. C. Genetics of nonsyndromic orofacial clefts. Cleft Palate Craniofac J 49, 73-91 (2012).

18. Carlson, J. C. et al. Identifying genetic sources of phenotypic heterogeneity in orofacial clefts by targeted sequencing. Birth Defects Res 109, 1030-1038 (2017). 
medRxiv preprint doi: https://doi.org/10.1101/2020.11.13.20231654; this version posted November 16, 2020. The copyright holder for this preprint (which was not certified by peer review) is the author/funder, who has granted medRxiv a license to display the preprint in perpetuity.

It is made available under a CC-BY-ND 4.0 International license .

19. Moreno Uribe, L. et al. A population-based study of effects of genetic loci on orofacial clefts. J Dent Res 96, 1322-1329 (2017).

20. Huang, L. et al. Genetic factors define CPO and CLO subtypes of nonsyndromic orofacial cleft. PLoS Genet 15, e1008357 (2019).

21. Howe, L. J. et al. Investigating the shared genetics of non-syndromic cleft lip/palate and facial morphology. PLoS Genet 14, e1007501 (2018).

22. Carlson, J. C. Methods for family-based designs in genetic epidemiology studies $\mathrm{PhD}$ thesis (University of Pittsburgh, 2017), 44-44.

23. Ray, D. \& Chatterjee, N. Effect of non-normality and low count variants on cross-phenotype association tests in GWAS. Eur J Hum Genet 28, 300-312 (2020).

24. Hackinger, S. \& Zeggini, E. Statistical methods to detect pleiotropy in human complex traits. Open Biol 7, 170125 (2017).

25. Fischer, S. T., Jiang, Y., Broadaway, K. A., Conneely, K. N. \& Epstein, M. P. Powerful and robust cross-phenotype association test for case-parent trios. Genet Epidemiol 42, 447-458 (2018).

26. Schaid, D. J. et al. Multivariate generalized linear model for genetic pleiotropy. Biostatistics 20, 111-128 (2019).

27. Ray, D. \& Chatterjee, N. A powerful method for pleiotropic analysis under composite null hypothesis identifies novel shared loci between type 2 diabetes and prostate cancer. bioRxiv (to appear in PLOS Genet), https://doi.org/10.1101/2020.04.11.037630 (2020).

28. Schutte, B. C., Saal, H. M., Goudy, S. \& Leslie, E. in GeneReviews (University of Washington, Seattle, 2014).

29. Dixon, M. J., Marazita, M. L., Beaty, T. H. \& Murray, J. C. Cleft lip and palate: understanding genetic and environmental influences. Nat Rev Genet 12, 167-178 (2011). 
30. Yu, Y. et al. Genome-wide analyses of non-syndromic cleft lip with palate identify 14 novel loci and genetic heterogeneity. Nat Commun 8, 1-11 (2017).

31. Van Rooij, I. A. et al. Non-syndromic cleft lip with or without cleft palate: genome-wide association study in europeans identifies a suggestive risk locus at 16p12.1 and supports SH3PXD2A as a clefting susceptibility gene. Genes 10, 1023 (2019).

32. Khramtsova, E. A., Davis, L. K. \& Stranger, B. E. The role of sex in the genomics of human complex traits. Nat Rev Genet 20, 173-190 (2019).

33. Harville, E. W., Wilcox, A. J., Lie, R. T., Vindenes, H. \& Åbyholm, F. Cleft lip and palate versus cleft lip only: are they distinct defects? Am J Epidemiol 162, 448-453 (2005).

34. Leslie, E. J. et al. Identification of functional variants for cleft lip with or without cleft palate in or near PAX7, FGFR2, and NOG by targeted sequencing of GWAS loci. Am J Hum Genet 96, 397-411(2015).

35. Rahimov, F. et al. Disruption of an AP-2 $\alpha$ binding site in an IRF6 enhancer is associated with cleft lip. Nat Genet 40, 1341-1347 (2008).

36. Sylvester, B. et al. A synonymous exonic splice silencer variant in IRF6 as a novel and cryptic cause of non-syndromic cleft lip and palate. Genes 11, 903 (2020).

37. Mostowska, A et al. Common variants in DLG1 locus are associated with non-syndromic cleft lip with or without cleft palate. Clin Genet 93, 784-793 (2018).

38. Bult, C. J., Blake, J. A., Smith, C. L., Kadin, J. A. \& Richardson, J. E. Mouse genome database (mgd) 2019. Nucleic Acids Res 47, D801-D806 (2019).

39. Wang, Y. et al. The 3D genome browser: a web-based browser for visualizing 3D genome organization and long-range chromatin interactions. Genome Biol 19, 1-12 (2018).

40. Zhang, R. et al. A unique methylation pattern co-segregates with neural tube defect statuses in Han Chinese pedigrees. Neurol Sci 38, 2153-2164 (2017). 
medRxiv preprint doi: https://doi.org/10.1101/2020.11.13.20231654; this version posted November 16, 2020. The copyright holder for this preprint (which was not certified by peer review) is the author/funder, who has granted medRxiv a license to display the preprint in perpetuity.

It is made available under a CC-BY-ND 4.0 International license .

41. Wilson, R. D. et al. Pre-conception folic acid and multivitamin supplementation for the primary and secondary prevention of neural tube defects and other folic acid-sensitive congenital anomalies. J Obstet Gynaecol Can 37, 534-549 (2015).

42. Wilderman, A., VanOudenhove, J., Kron, J., Noonan, J. P. \& Cotney, J. High-resolution epigenomic atlas of human embryonic craniofacial development. Cell Rep 23, 1581-1597 (2018).

43. Cejudo-Martin, P. et al. Genetic disruption of the $s h 3 p x d 2 a$ gene reveals an essential role in mouse development and the existence of a novel isoform of tks5. PLoS One 9, e107674 (2014).

44. Warner, D. R. et al. Micro RNA expression profiling of the developing murine upper lip. Dev Growth Differ 56, 434-447 (2014).

45. Duan, J. et al. A rare functional noncoding variant at the GWAS-implicated MIR137/MIR2682 locus might confer risk to schizophrenia and bipolar disorder. Am J Hum Genet 95, 744-753 (2014).

46. Bie, Y. \& Zhang, Z. RAB8A a new biomarker for endometrial cancer? World J Surg Oncol 12, $371(2014)$.

47. Marazita, M. L. et al. Genome scan, fine-mapping, and candidate gene analysis of non-syndromic cleft lip with or without cleft palate reveals phenotype-specific differences in linkage and association results. Hum Hered 68, 151-170 (2009).

48. Ludwig, K. et al. Strong association of variants around FOXE1 and orofacial clefting. J Dent Res 93, 376-381 (2014).

49. Beaty, T. H. et al. A genome-wide association study of cleft lip with and without cleft palate identifies risk variants near $M A F B$ and $A B C A 4$. Nat Genet 42, 525-529 (2010). 
medRxiv preprint doi: https://doi.org/10.1101/2020.11.13.20231654; this version posted November 16, 2020. The copyright holder for this preprint (which was not certified by peer review) is the author/funder, who has granted medRxiv a license to display the preprint in perpetuity. It is made available under a CC-BY-ND 4.0 International license .

50. Liu, H. et al. Identification of common non-coding variants at $1 \mathrm{p} 22$ that are functional for non-syndromic orofacial clefting. Nat Commun 8, 14759 (2017).

51. Liu, H. et al. Exome sequencing provides additional evidence for the involvement of ARHGAP29 in Mendelian orofacial clefting and extends the phenotypic spectrum to isolated cleft palate. Birth Defects Res 109, 27-37 (2017).

52. Leslie, E. J. et al. A multi-ethnic genome-wide association study identifies novel loci for non-syndromic cleft lip with or without cleft palate on 2p24. 2, 17q23 and 19q13. Hum Mol Genet 25, 2862-2872 (2016).

53. Skol, A. D., Scott, L. J., Abecasis, G. R. \& Boehnke, M. Joint analysis is more efficient than replication-based analysis for two-stage genome-wide association studies. Nat Genet 38, 209-213 (2006).

54. Das, S. et al. Next-generation genotype imputation service and methods. Nat Genet 48, 1284-1287 (2016).

55. Delaneau, O., Howie, B., Cox, A. J., Zagury, J.-F. \& Marchini, J. Haplotype estimation using sequencing reads. Am J Hum Genet 93, 687-696 (2013).

56. Taub, M. A., Schwender, H., Beaty, T. H., Louis, T. A. \& Ruczinski, I. Incorporating genotype uncertainties into the genotypic TDT for main effects and gene-environment interactions. Genet Epidemiol 36, 225-234 (2012).

57. Chang, C. C. et al. Second-generation PLINK: rising to the challenge of larger and richer datasets. GigaScience 4 (2015).

58. Howie, B. N., Donnelly, P. \& Marchini, J. A flexible and accurate genotype imputation method for the next generation of genome-wide association studies. PLoS Genet 5, e1000529 (2009). 
medRxiv preprint doi: https://doi.org/10.1101/2020.11.13.20231654; this version posted November 16, 2020. The copyright holder for this preprint (which was not certified by peer review) is the author/funder, who has granted medRxiv a license to display the preprint in perpetuity. It is made available under a CC-BY-ND 4.0 International license .

59. Schwender, H., Taub, M. A., Beaty, T. H., Marazita, M. L. \& Ruczinski, I. Rapid testing of SNPs and gene-environment interactions in case-parent trio data based on exact analytic parameter estimation. Biometrics 68, 766-773 (2012).

60. Schwender, H. et al. Detecting disease variants in case-parent trio studies using the bioconductor software package trio. Genet Epidemiol 38, 516-522 (2014).

61. R Core Team. R: A Language and Environment for Statistical Computing R Foundation for Statistical Computing (Vienna, Austria, 2018). https : / /www.R-project.org/.

62. Fallin, D., Beaty, T., Liang, K. Y. \& Chen, W. Power comparisons for genotypic vs. allelic TDT methods with $>2$ alleles. Genet Epidemiol 23, 458-461 (2002).

63. Spielman, R. S., McGinnis, R. E. \& Ewens, W. J. Transmission test for linkage disequilibrium: the insulin gene region and insulin-dependent diabetes mellitus (IDDM). Am J Hum Genet 52, 506-516 (1993).

64. Watanabe, K., Taskesen, E., Van Bochoven, A. \& Posthuma, D. Functional mapping and annotation of genetic associations with FUMA. Nat Commun 8, 1826 (2017).

65. Pruim, R. J. et al. LocusZoom: regional visualization of genome-wide association scan results. Bioinformatics 26, 2336-2337 (2010). 
medRxiv preprint doi: https://doi.org/10.1101/2020.11.13.20231654; this version posted November 16, 2020. The copyright holder for this preprint (which was not certified by peer review) is the author/funder, who has granted medRxiv a license to display the preprint in perpetuity. It is made available under a CC-BY-ND 4.0 International license .

Table 1: Association results for the most significant markers from the 9 loci showing statistical evidence of genetic overlap between CL/P and CP, along with 3 additional loci of genetic overlap between pairwise OFC subtypes. These loci were identified by PLACO at a suggestive threshold of $10^{-6}$. The genetic overlap analysis is based on all trios from both POFC and GENEVA for CL/P \& CP, or CL \& CP, or CLP \& CP, or CL \& CLP. The different types of novel genes are marked by $*$ or $\ddagger$. The "No. of trios" columns give the numbers of complete informative case-parent trios as used by the gTDT method in analyzing each OFC subgroup/subtype. 
(Caption in the previous page.)

\begin{tabular}{|c|c|c|c|c|c|c|c|c|c|c|c|}
\hline \multirow[b]{2}{*}{ Locus } & \multirow[b]{2}{*}{$\begin{array}{l}\text { Nearest } \\
\text { gene }\end{array}$} & \multirow[b]{2}{*}{ rsID } & \multirow[b]{2}{*}{$\begin{array}{r}\text { Position } \\
\text { (hg19) }\end{array}$} & \multirow{2}{*}{$\begin{array}{l}\text { Effect } \\
\text { allele } \\
\text { (freq.) }\end{array}$} & \multicolumn{3}{|c|}{$\mathrm{CL} / \mathrm{P}$} & \multicolumn{3}{|c|}{$\mathrm{CP}$} & \multirow{2}{*}{$\begin{array}{c}\text { CL/P \& CP } \\
\text { PLACO } \\
\text { p-value }\end{array}$} \\
\hline & & & & & $\begin{array}{l}\text { gTDT } \\
\text { p-value }\end{array}$ & $\begin{array}{c}\text { gTDT } \\
\text { RR }\end{array}$ & $\begin{array}{l}\text { No. of } \\
\text { trios }\end{array}$ & $\begin{array}{l}\text { gTDT } \\
\text { p-value }\end{array}$ & $\begin{array}{c}\text { gTDT } \\
\text { RR }\end{array}$ & $\begin{array}{c}\text { No. of } \\
\text { trios }\end{array}$ & \\
\hline $1 \mathrm{p} 36.13$ & $P A X 7^{\ddagger}$ & rs1339063 & 18989575 & $\mathrm{~T}(0.33)$ & $2.9 \times 10^{-8}$ & 1.27 & 1709 & $5.6 \times 10^{-3}$ & 0.77 & 385 & $6.9 \times 10^{-8}$ \\
\hline $1 \mathrm{q} 32.2$ & IRF6 & rs 72741048 & 209989092 & $\mathrm{~T}(0.34)$ & $4.0 \times 10^{-17}$ & 0.70 & 1799 & $2.8 \times 10^{-3}$ & 1.30 & 414 & $4.3 \times 10^{-12}$ \\
\hline $3 q 29$ & $D L G 1^{\ddagger}$ & rs 12632559 & 196803647 & $\mathrm{C}(0.42)$ & $1.7 \times 10^{-3}$ & 1.15 & 1521 & $2.1 \times 10^{-5}$ & 0.67 & 355 & $5.3 \times 10^{-7}$ \\
\hline $4 \mathrm{p} 13$ & $L I M C H 1^{\ddagger *}$ & rs9291207 & 41649103 & $\mathrm{C}(0.3)$ & $6.5 \times 10^{-5}$ & 0.84 & 1611 & $8.0 \times 10^{-4}$ & 1.38 & 347 & $5.0 \times 10^{-7}$ \\
\hline $4 q 21.1$ & SHROOM $3^{\ddagger}$ & rs4422437 & 77514866 & $\mathrm{G}(0.33)$ & $6.8 \times 10^{-7}$ & 1.23 & 1841 & $9.4 \times 10^{-3}$ & 0.80 & 431 & $8.1 \times 10^{-7}$ \\
\hline $9 q 22.33$ & FOXE1 & rs 12347191 & 100619719 & $\mathrm{C}(0.25)$ & $8.9 \times 10^{-6}$ & 0.81 & 1526 & $1.1 \times 10^{-3}$ & 0.73 & 351 & $1.7 \times 10^{-7}$ \\
\hline $17 q 22$ & $N O G$ & rs4794658 & 54766218 & $\mathrm{~T}(0.36)$ & $1.9 \times 10^{-6}$ & 1.21 & 1951 & $1.1 \times 10^{-3}$ & 0.75 & 407 & $6.0 \times 10^{-8}$ \\
\hline $19 \mathrm{p} 13.12$ & $R A B 8 A^{\ddagger *}$ & rs7252188 & 16228701 & $\mathrm{~A}(0.39)$ & $3.2 \times 10^{-3}$ & 0.89 & 1844 & $9.2 \times 10^{-6}$ & 0.69 & 455 & $6.8 \times 10^{-7}$ \\
\hline \multirow[t]{2}{*}{$20 \mathrm{q} 12$} & $M A F B^{\ddagger}$ & rs6016392 & 39246610 & $\mathrm{~A}(0.5)$ & $8.3 \times 10^{-10}$ & 1.27 & 2004 & $3.9 \times 10^{-2}$ & 1.18 & 452 & $9.9 \times 10^{-7}$ \\
\hline & & & & & \multicolumn{3}{|c|}{$\mathrm{CL}$} & \multicolumn{3}{|c|}{$\mathrm{CP}$} & $\mathrm{CL} \& \mathrm{CP}$ \\
\hline \multirow[t]{2}{*}{$18 \mathrm{q} 12.1$} & $\operatorname{MIR} 302 F^{\ddagger *}$ & rs 11083400 & 28056959 & $\mathrm{~T}(0.45)$ & $3.1 \times 10^{-3}$ & 0.79 & 484 & $1.6 \times 10^{-5}$ & 1.45 & 424 & $6.2 \times 10^{-7}$ \\
\hline & & & & & \multicolumn{3}{|c|}{ CLP } & \multicolumn{3}{|c|}{$\mathrm{CP}$} & CLP \& CP \\
\hline \multirow[t]{2}{*}{$10 \mathrm{q} 24.33$} & $S H 3 P X D 2 A^{\ddagger}$ & rs11191818 & 105591779 & $\mathrm{G}(0.31)$ & $2.7 \times 10^{-4}$ & 0.82 & 1102 & $5.1 \times 10^{-4}$ & 1.40 & 341 & $9.2 \times 10^{-7}$ \\
\hline & & & & & \multicolumn{3}{|c|}{ CL } & \multicolumn{3}{|c|}{ CLP } & CL \& CLP \\
\hline $1 \mathrm{p} 21.3$ & $M I R 137 H G^{\ddagger *}$ & $\mathrm{rs} 2802532$ & 98528830 & $\mathrm{C}(0.09)$ & $7.3 \times 10^{-5}$ & 0.59 & 217 & $3.5 \times 10^{-5}$ & 1.41 & 562 & $2.2 \times 10^{-8}$ \\
\hline
\end{tabular}

${ }_{\ddagger}^{\ddagger}$ Genes that have not previously been suggested as regions of genetic overlap between OFC subgroups in linkage or association studies.

*Genes that have not been previously implicated in OFC genetics.

Abbreviations: Chr, chromosome; CL/P, cleft lip with or without palate; CP, cleft palate; Freq., frequency; gTDT, genotypic transmission disequilibrium test; OFC, orofacial cleft; PLACO, pleiotropic analysis under composite null hypothesis; RR, relative risk (with respect to the reported effect allele) 


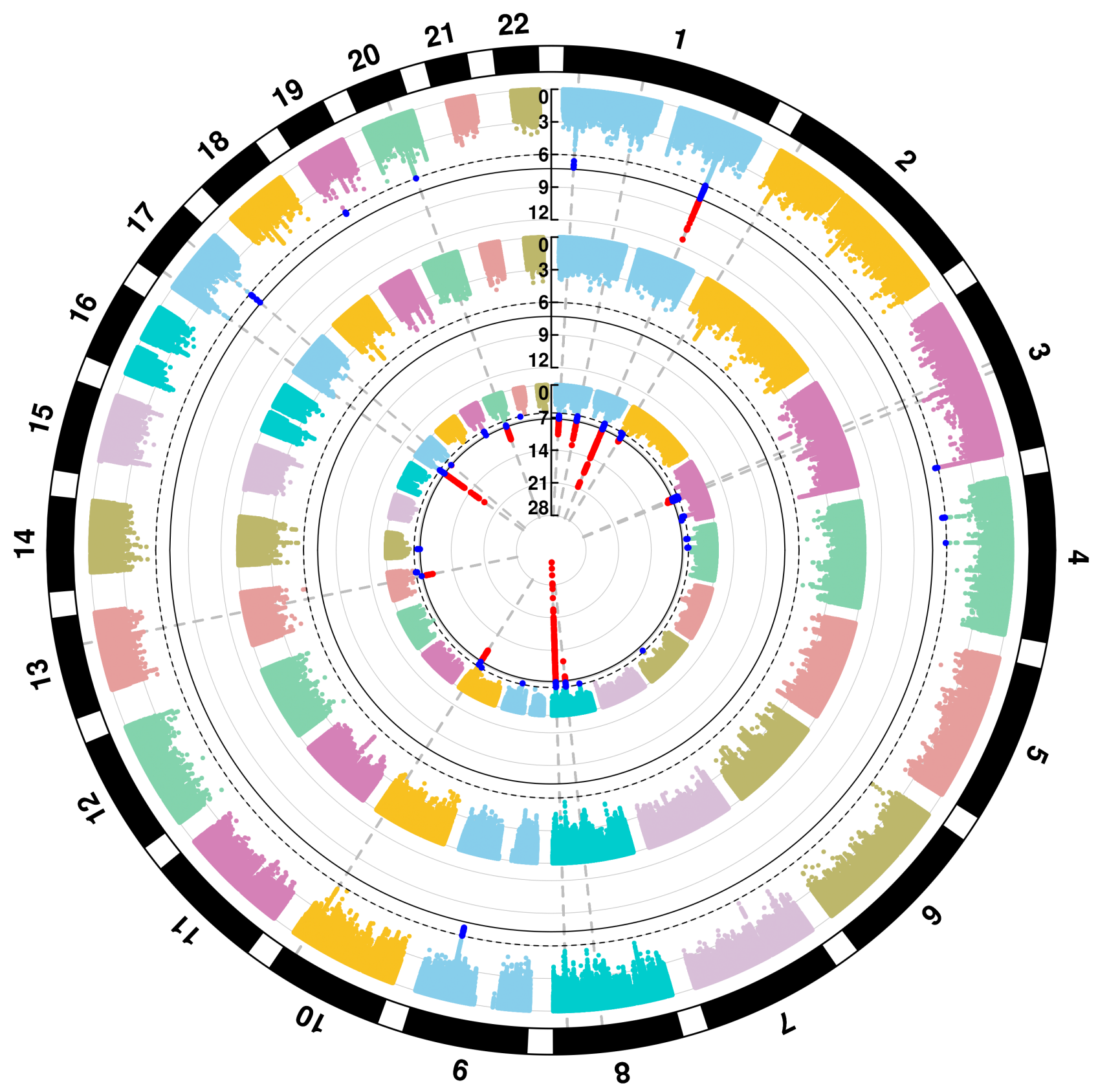

Figure 1: Manhattan plots for genome-wide analyses of OFC subgroups. The plots are of negative log-transformed p-values from the analysis of cleft lip with or without cleft palate (CL/P, innermost circle), of cleft palate (CP, intermediate circle), and of genetic overlap between CL/P \& CP using PLACO (outermost circle). The chromosome numbers 1-22 are indicated along the outermost circumference. Solid black and dashed black circular lines are used in all plots to indicate the conventional genome-wide significance threshold $5 \times 10^{-8}$ and a less stringent suggestive threshold $10^{-6}$ respectively. The variants exceeding the genome-wide and the liberal thresholds are respectively colored in red and bright blue. 
medRxiv preprint doi: https://doi.org/10.1101/2020.11.13.20231654; this version posted November 16, 2020. The copyright holder for this preprint (which was not certified by peer review) is the author/funder, who has granted medRxiv a license to display the preprint in perpetuity.

It is made available under a CC-BY-ND 4.0 International license .
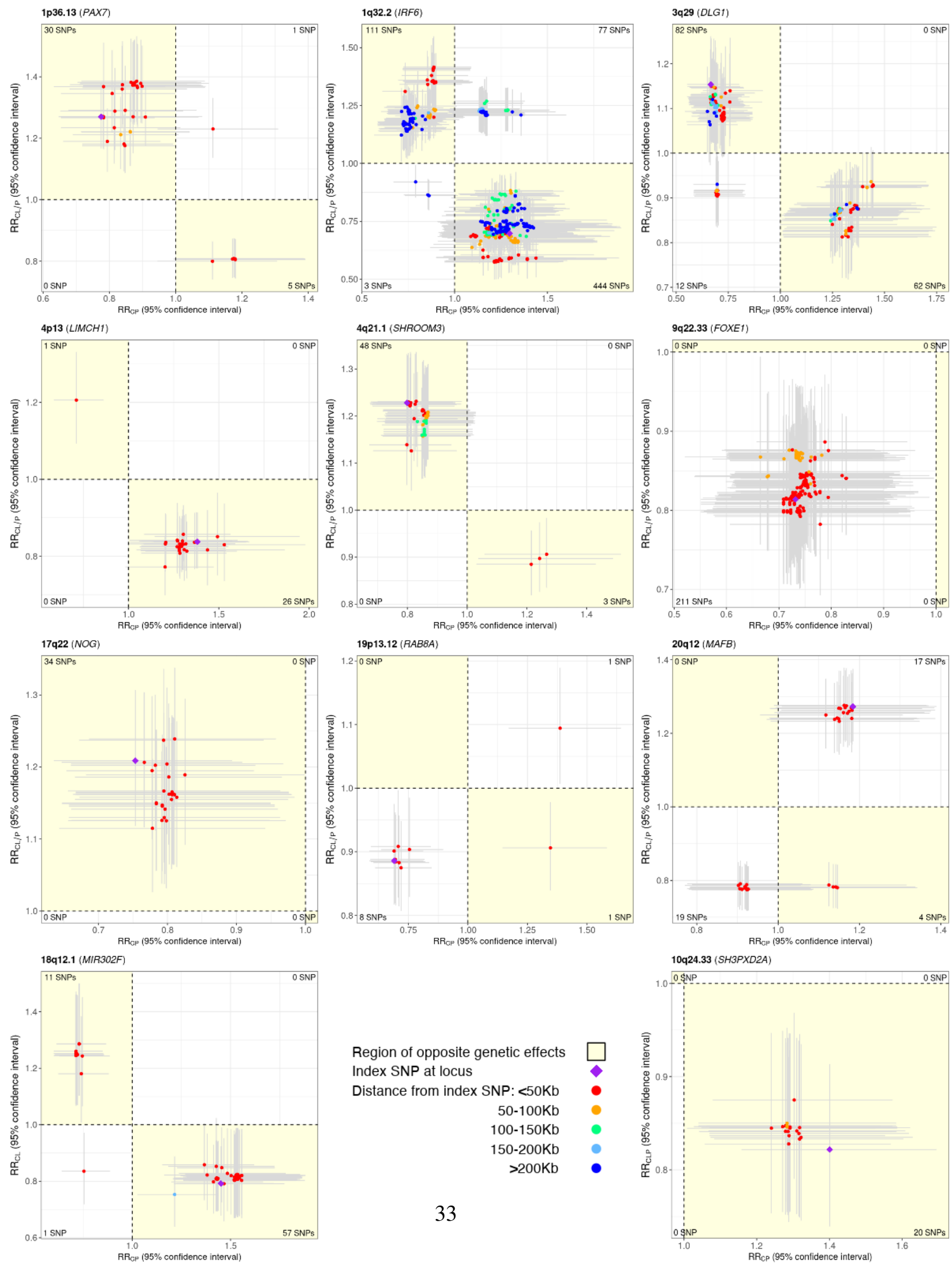

Region of opposite genetic effects Index SNP at locus

Distance from index $\mathrm{SN}$

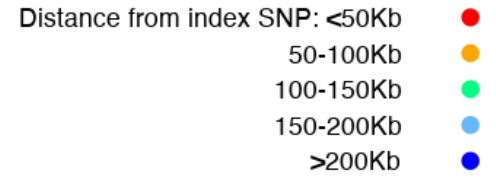

33

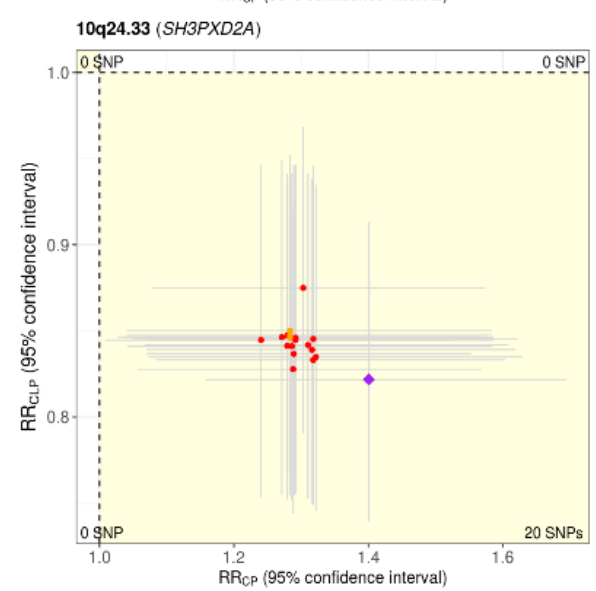

(Caption in the next page.) 
Figure 2: Scatter plot of relative risk (RR) estimates, along with corresponding $95 \%$ confidence intervals (CIs), for variants in the 9 loci showing statistical evidence of genetic overlap between CL/P \& CP, along with 2 additional loci of genetic overlap between component OFC subtypes. RR estimates are color annotated based on distance of SNPs from the index/lead SNP. LD-based color annotation is not used since these RR estimates are from multi-ethnic analyses and consequently, there is no unique LD between SNPs. Horizontal (vertical) error bar around each RR estimate corresponds to the 95\% CI for the OFC subgroup represented on the x-axis (y-axis). The region depicting opposite genetic effects of SNPs for the 2 OFC subgroups is shaded in yellow. The number of SNPs in each quadrant is printed in the corresponding corner of the plot. The SNPs plotted here are in $\pm 500 \mathrm{~Kb}$ radius and in LD $r^{2}>0.2$ with the index/lead SNP, and further screened out SNPs with PLACO p-value $>10^{-3}$ from the respective genetic overlap analysis. These plots show genetically distinct etiology of CL/P and CP at 6 loci (i.e., overlapping genetic etiology with opposite effects), and of the component OFC subtypes at 2 other loci as depicted by the SNPs in the yellow shaded region. 
medRxiv preprint doi: https://doi.org/10.1101/2020.11.13.20231654; this version posted November 16, 2020. The copyright holder for this preprint (which was not certified by peer review) is the author/funder, who has granted medRxiv a license to display the preprint in perpetuity.

It is made available under a CC-BY-ND 4.0 International license .

a

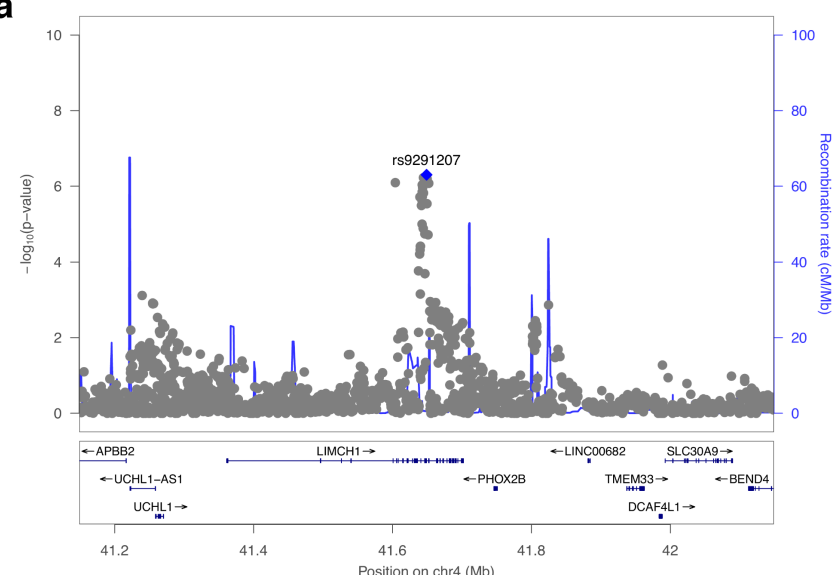

C

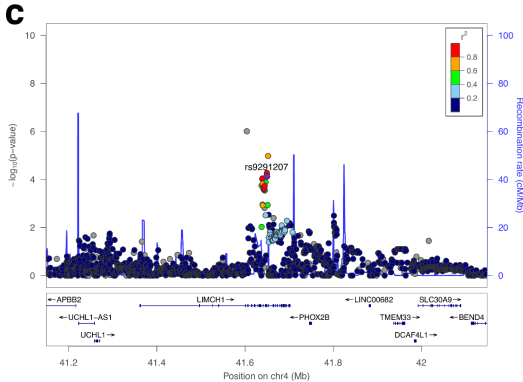

f

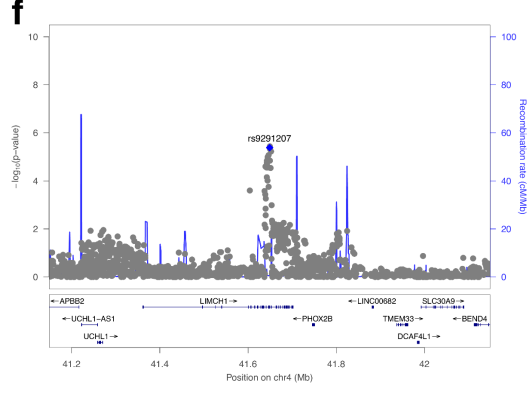

d

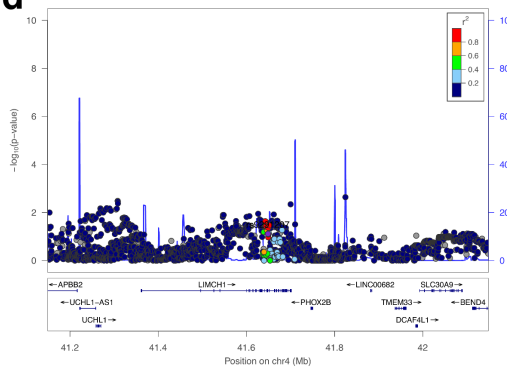

g

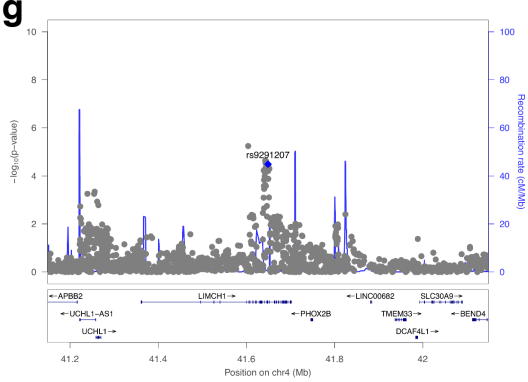

b rs9291207 (LIMCH1); effect allele $=\mathrm{C}$

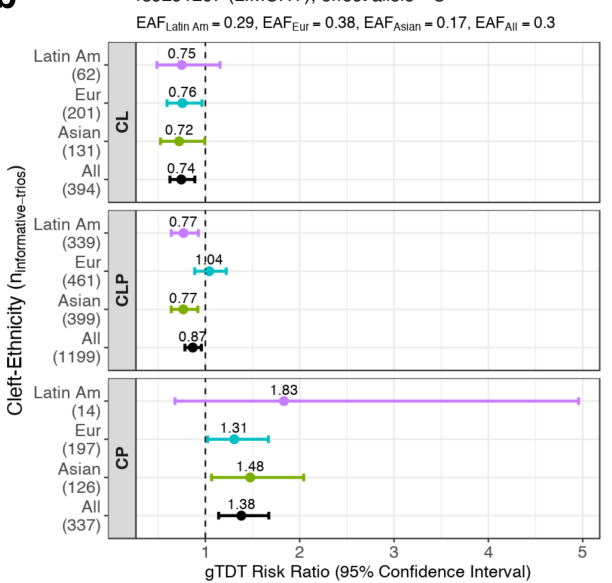

e

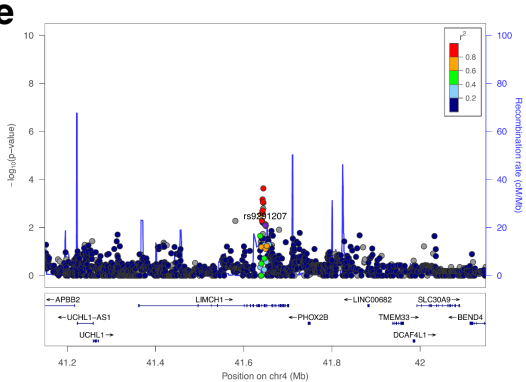

$\mathbf{h}$

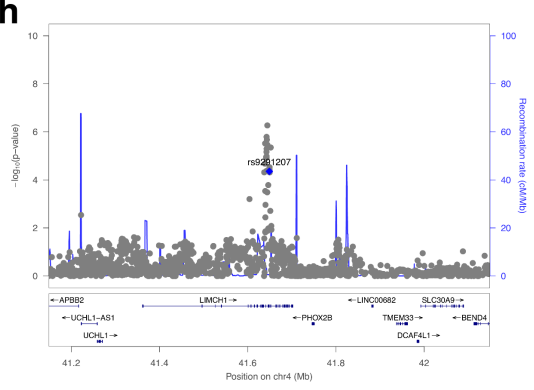

Figure 3: Regional association plots for 4 p13 (LIMCH1) identified as a region of genetic overlap between CL/P \& CP. LocusZoom plots focus on PLACO analysis of (a) CL/P \& CP (multi-ethnic), (c) CL/P \& CP in Asian ancestry, (d) CL/P \& CP in European ancestry, (e) CL/P \& CP in Latin American ancestry, (f) CL \& CP (multi-ethnic), (g) CLP \& CP (multi-ethnic), (h) CL \& CLP (multi-ethnic). The blue or purple diamond represents the most strongly associated SNP in the region showing evidence of genetic overlap. For stratified analyses across racial/ethnic groups, the colors of the SNPs represent their LD with the lead SNP (the most strongly associated SNP from panel a), as shown in the color legend. For combined multi-ethnic analyses, there is no unique LD between SNPs and hence no color has been used. Panel b shows relative risk estimates of the lead SNP and their 95\% confidence intervals as obtained from the gTDT analyses. 


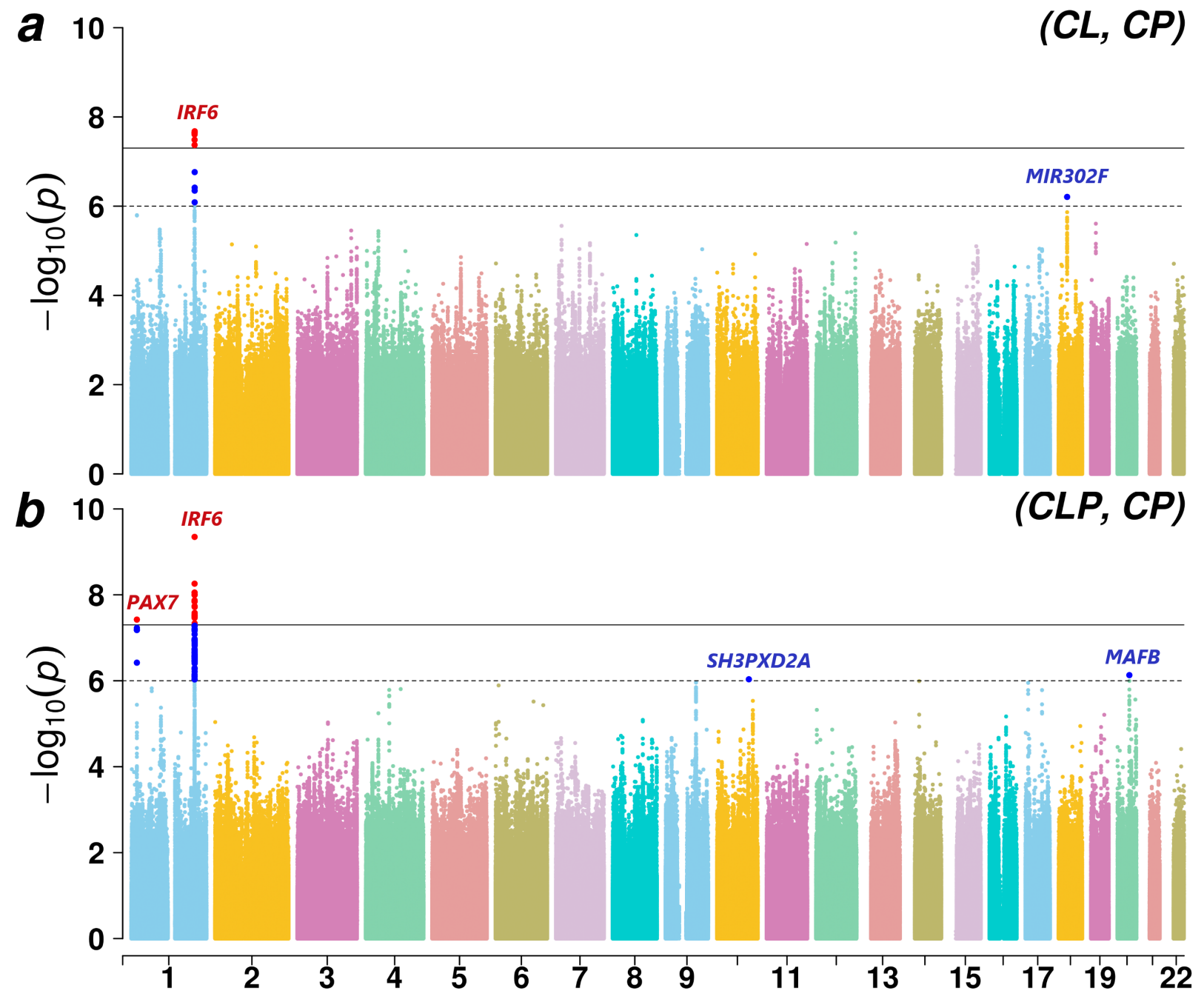

Figure 4: Manhattan plots for genome-wide analyses of genetic overlap between pairs of OFC subtypes: CL

\& CP, and CLP \& CP. The chromosome numbers 1-22 are indicated along the x-axis. Solid black and dashed black lines are used in both plots to indicate the conventional genome-wide significance threshold $5 \times 10^{-8}$ and a less stringent suggestive threshold $10^{-6}$ respectively. The variants exceeding the genome-wide and the liberal thresholds are respectively colored in red and bright blue. 


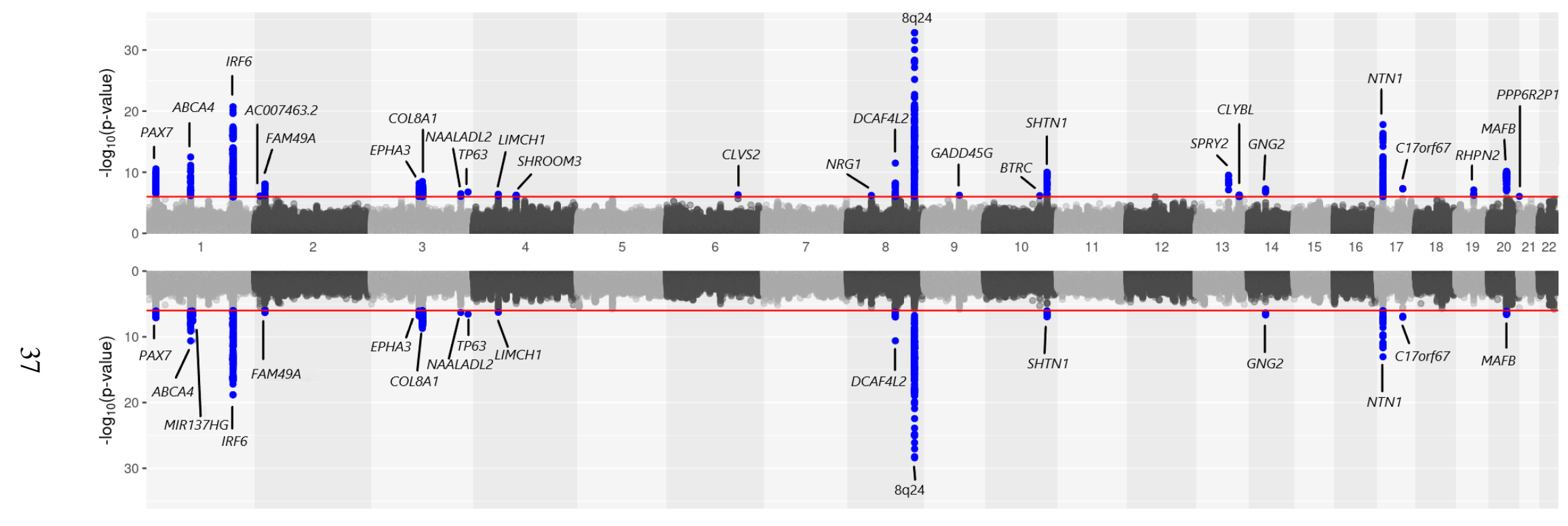

Figure 5: Mirrored Manhattan plot of genome-wide analyses of CL/P, and CL \& CLP. Results from genetic associations of CL/P using the gTDT are shown in the upper panel. Results from shared genetic associations between CL \& CLP using PLACO are shown in the lower panel. The red horizontal lines in the two panels indicate a suggestive significance threshold of $10^{-6}$. 